Acta Veterinaria Hungarica 62 (3), pp. 422-427 (2014)

DOI: 10.1556/AVet.2014.011

First published online 19 July 2014

\title{
NON-PRIMATE HEPACIVIRUS INFECTION WITH APPARENT HEPATITIS IN A HORSE - SHORT COMMUNICATION
}

\author{
Gábor REUTER $^{1 *}$, Norbert MAZA ${ }^{2}$, Péter PANKOvics ${ }^{1}$ and Ákos BoRos ${ }^{1}$ \\ ${ }^{1}$ Regional Laboratory of Virology, National Reference Laboratory of Gastroenteric \\ Viruses, ÁNTSZ Regional Institute of State Public Health Service, Szabadság út 7, \\ H-7623 Pécs, Hungary; ${ }^{2}$ Directorate of Food Chain Safety and Animal Health, \\ Government Office for Baranya County, Pécs, Hungary
}

(Received 24 October 2013; accepted 12 March 2014)

\begin{abstract}
Non-primate hepacivirus (NPHV) is a recently identified hepacivirus (family Flaviviridae) in dog and horse; however, the disease associations remain unknown. This study reports the detection of natural NPHV infection in a horse with apparent hepatitis, liver damage and high-level viraemia. NPHV could be hepatotropic and associated with hepatitis in horses.
\end{abstract}

Key words: Non-primate hepacivirus, hepacivirus, Flaviviridae, hepatitis, viraemia, horse

The genus Hepacivirus, one of the four genera in the family Flaviviridae, comprises the assigned prototype species, hepatitis $\mathrm{C}$ virus (HCV) and an unassigned GB virus B (GBV-B) (Simmonds et al., 2012). Although the evolutionary origin of human hepatitis C virus remains undetermined (Simmonds, 2013), a close non-primate homologue of this virus (now called non-primate hepacivirus, NPHV) has been recently identified in dogs in the USA (Kapoor et al., 2011) and in horses in the USA (Burbelo et al., 2012), UK (Lyons et al., 2012) and Germany (Drexler et al., 2013). Canine hepacivirus (CHV) was detected in the respiratory tract with a potential association with respiratory disease (Kapoor et al., 2011). NPHV RNA was detected in $8(7.8 \%)$ of 103,3 of $142(2.1 \%)$ and 7 of $210(3.3 \%)$ plasma samples collected from healthy horses in the USA, UK and Germany (Burbelo et al., 2012; Lyons et al., 2012; Drexler et al., 2013). In addition, results of a novel serological test for antibodies to the conserved nonstructural protein 3 (NS3) showed a seropositivity of 35\% among these horses in the USA (Burbelo et al., 2012). Disease associations of NPHV in horses remain unknown. Clinical records of the three infected horses in the UK failed to identify evidence of hepatitis or systemic disease; however, a followed animal was viraemic during the 5-month follow-up period (Lyons et al., 2012).

*Corresponding author; E-mail: reuter.gabor@ddr.antsz.hu; Phone: 0036 (72) 514-979; Fax: 0036 (72) 514-949 
This study reports the detection of natural NPHV infection in a horse with apparent hepatitis in Hungary. NPHV could be associated with hepatitis in horses.

\section{Materials and methods}

Blood samples were collected on 1 March, 2 April, 26 May and 13 August 2013 from an 11-year-old male domestic horse (DH1) living at a family stall in Baranya County, Hungary. Liver function tests (Table 1) were done for biochemical confirmation and follow-up of hepatitis and hepatic insufficiency. Blood samples were separated by centrifugation and frozen at $-80^{\circ} \mathrm{C}$ until reverse transcription-PCR (RT-PCR) testing. Specific primer pairs (EQNS5BIS/ EQNS5BOIAS) were used for detection of nonstructural protein NS5B of NPHV by RT-PCR described previously (Lyons et al., 2012) (Table 1). Specific reverse or forward primers were designed based on the available NPHV sequences to determine the complete viral genome by RT-PCR (Table 1). Specific primer pairs were also used for excluding two novel equine pegivirus (family Flaviviridae) infections [Theiler's disease-associated virus (TDAV) and equine pegivirus $(\mathrm{EPgV})]$ in horse by RT-PCR published recently (Chandriani et al., 2013; Kapoor et al., 2013). PCR products were sequenced directly (BigDye Reaction Kit, Applied Biosystems, Warrington, UK) using the PCR primers and run on an automated sequencer (ABI PRISM 310 Genetic Analyzer, Applied Biosystems, Stafford, Texas, USA).

Table 1

Biochemical characteristics, non-primate hepacivirus (NPHV) detection and quantification in serum pairs collected from a domestic horse (DH1) with clinical jaundice in acute (sera 1 and 2) and convalescent (sera 3 and 4 ) phases

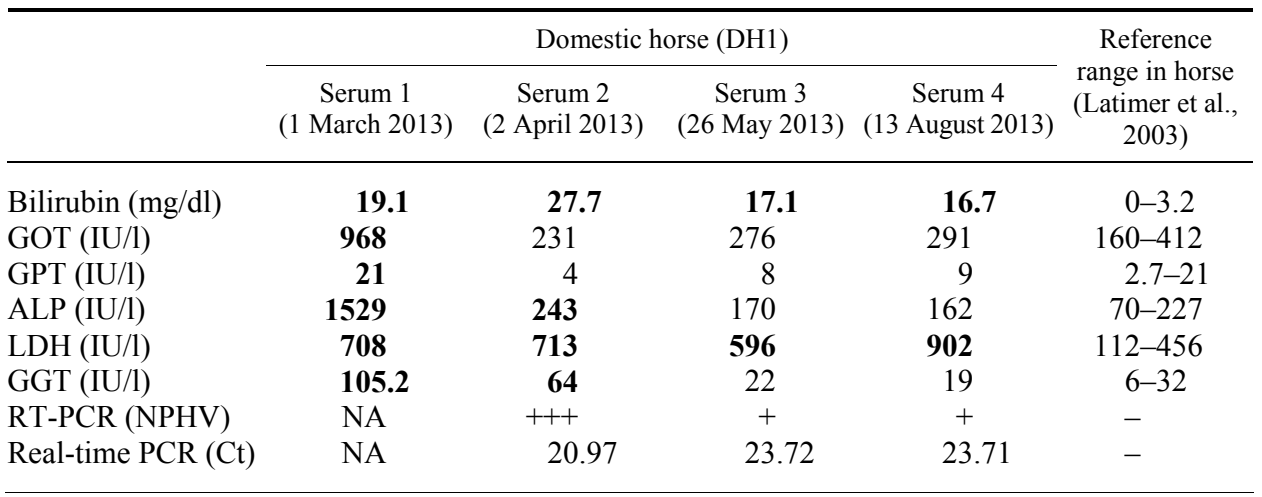

Elevated liver function test results are indicated with bold letters. GOT: glutamate-oxaloacetate transaminase; GPT: glutamic-pyruvic transaminase; ALP: alkaline phosphatase; LDH: lactate dehydrogenase; GGT: gamma-glutamyl transferase; RT-PCR: reverse transcription-polymerase chain reaction; $\mathrm{Ct}$ : crossing threshold; NA: not available 
Reference sequences were obtained from GenBank and aligned using Clustal X, then similarity calculations were performed by GeneDoc. Phylogenetic tree of the amino acid alignment of nonstructural protein 3 (NS3) was created using the neighbour-joining method based on the Jones-Taylor-Thornton matrix-based model of MEGA5 (Tamura et al., 2011). RNA quantification relied on primers (NPHV-5UTR-F: 5'-CCACGGTCTGATAGGATGCTT corresponding to nt 329 to 349 of JQ434002; NPHV-5UTR-R: 5'-GCCCGCGGATGGCCT TCTT corresponding to nt 523 to 541 of JQ434002) designed for the highly conserved 5'-untranslated region (UTR) using real-time PCR assay (LightCycler FastStart DNA Master SYBR Green I, Roche, Mannheim, Germany). The complete genome sequence of NPHV found in the Hungarian horse has been submitted to GenBank under the accession number KF177391.

\section{Results}

On 1 March 2013, during a veterinary visit, a domestic horse (DH1) showing severe jaundice (conjunctival icterus) of unknown origin was found in a family stall in Baranya County, Hungary. DH1 had no history of jaundice or intravenous administration of transfusion or passive immunisations in the previous year; however, it was vaccinated yearly with inactivated influenza/tetanus (Duvaxyn IE-T Plus, Fort Dodge AH) and West Nile virus (Duvaxyn WNV, Fort Dodge $\mathrm{AH})$ vaccines. Liver function tests confirmed hepatitis and hepatic insufficiency (Table 1). Routine microscopic examination of a blood smear was negative for Babesia, microfilariae, Ehrlichia, Trypanosoma and Anaplasma. Microagglutination (OIE MM 2008:2.1.9.B.2.a) and agar immunodiffusion (OIE MM 2008:2.5.6.B.2.a) tests were negative for leptospirosis (Leptospira hardjo, $L$. grippotyphosa, L. icterohaemorrhagiae, L. tarassovi and L. pomona) and equine infectious anaemia virus (EIAV) infections, respectively (http://www.oie.int/ international-standard-setting/terrestrial-manual/access-online). When the possibility of NPHV infection was revealed, another blood sample (serum 2) was collected and tested strongly positive for NPHV by conventional RT-PCR. For a follow-up study, blood samples were collected again on 26 May and 13 August 2013, when the intensity of the jaundice had clinically decreased. These sera showed a lower level of positivity for NPHV by RT-PCR (Table 1). NPHV viral loads were parallel measured in the sera by real-time PCR (Table 1). The crossing threshold $(\mathrm{Ct})$ was considerably lower in the acute than in the convalescent stage, indicating a higher amount of NPHV during the clinical phase (Table 1). Complete NPHV viral genome (9219 nt + polyA) of strain NPHV-DH1/2013/HUN (KF177391) was characterised from serum 2. The Hungarian NPHV clustered phylogenetically with the previously described equine hepaciviruses. Figure 1 shows the phylogenetic analysis of NPHV-DH1/2013/HUN and hepaciviruses 
based upon the amino acid sequences of NS3 (nt 3545 to 5523 in the EpgV; KC410872) (Fig. 1). Compared to the available complete genome sequences of NPHV $(\mathrm{n}=10)$, the DH1 nucleotide differences $(10-17 \%)$ represented mostly synonymous mutations, resulting in amino acid distances of 3-6\%.

Sera $2-4$ of DH1 were negative for Theiler's disease-associated virus (TDAV) and equine pegivirus (EPgV) by RT-PCR.

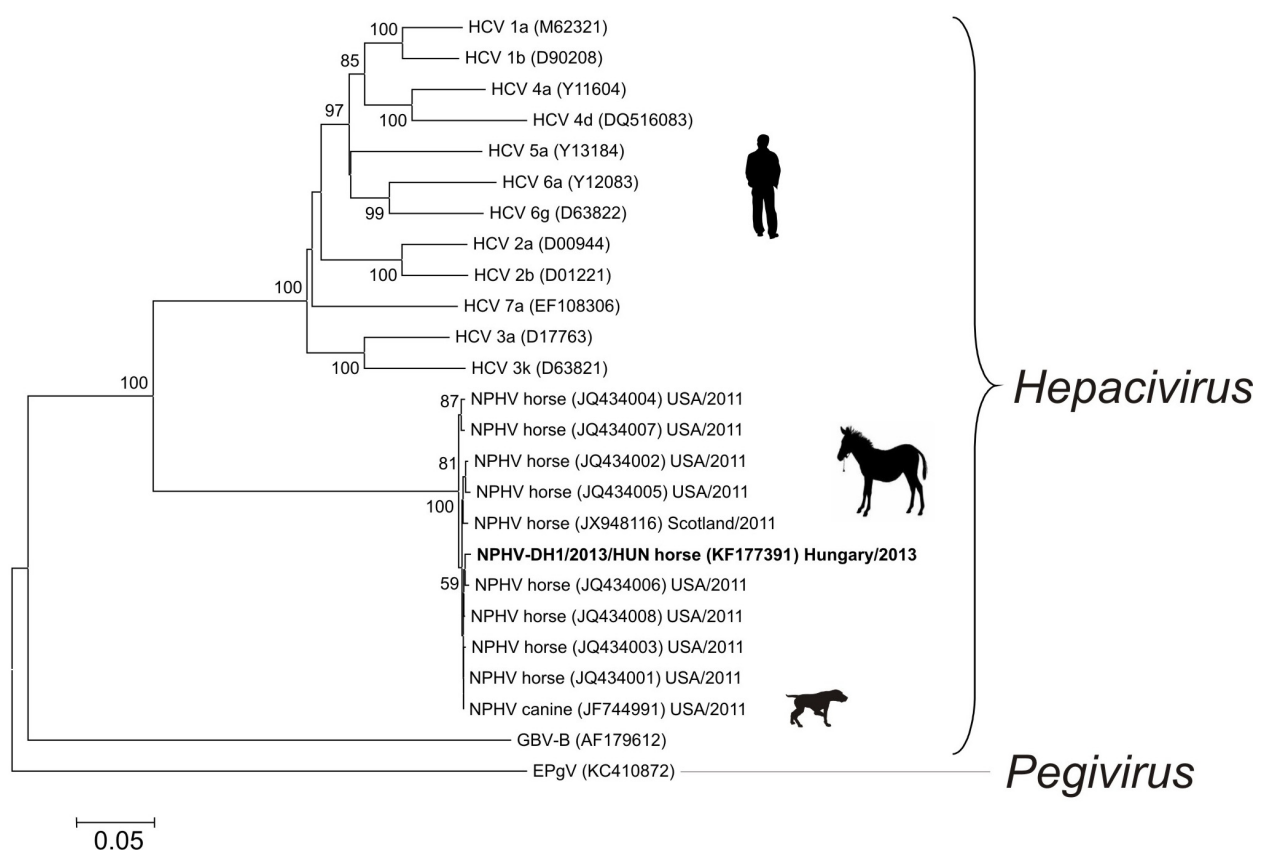

Fig. 1. Phylogenetic analysis (neighbour-joining method, MEGA version 5.1) of NPHVDH1/2013/HUN (KF177391; bold letters) and other representative hepaciviruses including the available NPHV genomes in GenBank based upon the amino acid sequences of nonstructural protein 3 (NS3) (from nt 3545 to nt 5523 in the EpgV genome, KC410872). HCV: hepatitis C virus; NPHV: non-primate hepacivirus; GBV-B: GB virus B; $\mathrm{EPgV}$ : equine pegivirus. Equine pegivirus was used as outgroup to root the tree

\section{Discussion}

Although the recently identified NPHV is a non-primate homologue of hepatitis $\mathrm{C}$ virus causing hepatitis in humans, the disease associations of NPHV in horses remain unknown. This study reports the detection of a natural NPHV infection in a horse with apparent hepatitis. Both the clinical signs and the liver function tests provided a clear indication of liver damage. The first-round PCR was already positive for the detection of NPHV, and therefore the nested-PCR 
assay was not necessary as described by Lyons et al. (2012), which indicates a high amount of viral nucleic acid in sera. In addition, the high level of viraemia was also confirmed by the low level of crossing threshold $(\mathrm{Ct})$ by real-time PCR. The laboratory test results (viral nucleic acid level, liver function test) changed parallel to the clinical findings (intensity of jaundice) during the follow-up investigation. Although sera obtained from DH1 4.5 months apart were viraemic, indicating persistent infection, during clinical recovery the viral nucleic acid level was lower and most of the liver function test results returned to the normal reference ranges. Exclusion of the presence of two novel pegiviruses (TDAV and $\mathrm{EPgV}$ ) in family Flaviviridae, which are likely (TDAV) and possibly (EPgV) associated with liver infection and disease in horses (Chandriani et al., 2013; Kapoor et al., 2013) strengthen the case for a link between NPHV and liver disease.

Although the source and the time of NPHV infection (acute or a reactivation of a chronic, clinically inapparent infection?) remains unknown, non-primate hepacivirus could be hepatotropic and associated with symptomatic hepatitis in horses.

\section{Acknowledgements}

This work was supported by a grant from the Hungarian Scientific Research Fund (OTKA, K83013). G. R. was supported by the János Bolyai Research Scholarship of the Hungarian Academy of Sciences. We thank Dr. Tamás Köszegi (University of Pécs, Pécs, Hungary) and Dr. Csaba Nemes (National Food Chain Safety Office, Kaposvár, Hungary) for their help with the liver function tests and the routine microbiological diagnostic tests, respectively.

\section{References}

Burbelo, P. D., Dubovi, E. J., Simmonds, P., Medina, J. L., Henriquez, J. A., Mishra, N., Wagner, J., Tokarz, R., Cullen, J. M., Ladarola, M. J., Rice, C. M., Lipkin, J. M. and Kapoor, A. (2012): Serology-enabled discovery of genetically diverse hepaciviruses in a new host. J. Virol. 86, 6171-6178.

Chandriani, S., Skewes-Cox, P., Zhong, W., Ganem, D. E., Divers, T. J., Van Blaricum, A. J., Tennat, B. C. and Kistler, A. L. (2013): Identification of a previously undescribed divergent virus from the Flaviviridae family in an outbreak of equine serum hepatitis. Proc. Natl Acad. Sci. USA 110, 1407-1415.

Drexler, J. F., Corman, V. M., Müller, M. A., Lukashev, A. N., Gmyl, A., Coutard, B., Adam, A., Ritz, D., Leijten, L. M., van Riel, D., Kallies, R., Klose, S. M., Gloza-Rausch, F., Binger, T., Annan, A., Adu-Sarkodie, Y., Oppong, S., Bourgarel, M., Rupp, D., Hoffmann, B., Schlegel, M., Kümmerer, B. M., Krüger, D. H., Schmidt-Chanasit, J., Setién, A. A., Cottontail, V. M., Hemachudha, T., Wacharapluesadee, S., Osterrieder, K., Bartenschlager, R., Matthee, S., Beer, M., Kuiken, T., Reusken, C., Leroy, E. M., Ulrich, R. G. and Drosten, C. (2013): Evidence of novel hepaciviruses in rodents. PLoS Pathog. 9, e1003438.

Kapoor, A., Simmonds, P., Cullen, J. M., Scheel, T. K. H., Medina, J. L., Giannitti, F., Nishiuchi, E., Brock, K. V., Burbelo, P. D., Rice, C. M. and Lipkin, W. I. (2013): Identification of a pegivirus (GB virus-like virus) that infects horses. J. Virol. 87, 7185-7190. 
Kapoor, A., Simmonds, P., Gerold, G., Qaisar, N., Jain, K., Henriquez, J. A., Firth, C., Hirschberg, D. L., Rice, C. M., Shields, S. and Lipkin, W. I. (2011): Characterization of a canine homolog of hepatitis C virus. Proc. Natl Acad. Sci. USA 108, 11608-11613.

Latimer, K. S., Mahaffey, E. A. and Prasse, K. W. (2003): Duncan and Prasse's Veterinary Laboratory Medicine: Clinical Pathology, 4th edition. Wiley-Blackwell, Ames, IA, USA.

Lyons, S., Kapoor, A., Sharp, C., Schneider, B. S., Wolfe, N. D., Culshaw, G., Corcoran, B., McGorum, B. C. and Simmonds, P. (2012): Nonprimate hepaciviruses in domestic horses, United Kingdom. Emerg. Infect. Dis. 18, 1976-1982.

Simmonds, P. (2013): The origin of hepatitis C virus. Curr. Top. Microbiol. Immunol. 369, 1-15.

Simmonds, P., Becher, P., Collett, M. S., Gould, E. A., Heinz, F. X., Meyers, G., Monath, T., Pletnev, A., Rice, C. M., Stiasny, K., Thiel, H. J., Weiner, A. and Bukh, J. (2012): Hepacivirus. In: King, A. M. Q., Adams, M. J., Carstens, E. B. and Lefkowitz, E. J. (eds) Virus Taxonomy: Classification and Nomenclature of Viruses: Ninth Report of the International Committee on Taxonomy of Viruses, Elsevier, San Diego. pp. 1014-1020.

Tamura, K., Peterson, D., Peterson, N., Stecher, G., Nei, M. and Kumar, S. (2011): MEGA5: Molecular Evolutionary Genetics Analysis using maximum likelihood, evolutionary distance, and maximum parsimony methods. Mol. Biol. Evol. 28, 2731-2739. 